\title{
Direct Measurement of Gaseous Formaldehyde from Food with a Fiber-Optic Biochemical Gas Sensor (Bio-sniffer)
}

\author{
Koji Toma, ${ }^{1}$ Kumiko Miyajima, ${ }^{1,2}$ Shin-ichi Sawada, ${ }^{3}$ Takahiro Arakawa, ${ }^{1}$ \\ Hiroyuki Kudo, ${ }^{1 \dagger}$ Kazunari Akiyoshi, ${ }^{3}$ and Kohji Mitsubayashi ${ }^{1 *}$ \\ ${ }^{1}$ Department of Biomedical Devices and Instrumentation, Institute of Biomaterials and Bioengineering, \\ Tokyo Medical and Dental University, 2-3-10 Kanda-Surugadai, Chiyoda, Tokyo 101-0062, Japan \\ ${ }^{2}$ Japan Society for the Promotion of Science, 5-3-1 Kojimachi, Chiyoda, Tokyo 102-0083, Japan \\ ${ }^{3}$ Graduate School of Engineering, Kyoto University, \\ 1-5-45 Kyotodaigaku-Katsura, Nishikyo, Kyoto 615-8510, Japan
}

(Received March 14, 2016; accepted June 13, 2016)

Keywords: enzyme, fluorometry, gas sensor, formaldehyde, fiber-optic, food sample

In this work, we assessed the ability of a fiber-optic biochemical gas sensor (bio-sniffer) to directly and nondestructively measure gaseous formaldehyde (FA) released from food. In the biosniffer, formaldehyde dehydrogenase (FALDH) was immobilized in a hydrophilic fluoropolymer membrane in which an enzymatic reaction producing nicotinamide adenine dinucleotide (NADH) occurred. The resultant NADH was excited with ultraviolet (UV) light irradiated from the optical fiber end in the bio-sniffer and the emitted fluorescence was coupled back to the fiber end and detected. In experiments to characterize the bio-sniffer using standard gaseous FA samples, the capability of continuous measurement (a coefficient of variation of $3.4 \%$ for five successive measurements) and high sensitivity (a limit of detection of $1.1 \mathrm{ppb}$ ) were validated. Then a demonstration of direct gaseous FA from food was carried out using Shiitake mushrooms (Lentinula edodes). Owing to the use of the enzyme, the bio-sniffer was able to selectively measure FA, and clear differences in FA concentrations were observed depending on the state of the mushrooms, i.e., dried, rehydrated with deionized water or with $\gamma$-GTPase inhibitor. These results indicated that the bio-sniffer allowed the direct monitoring of FA naturally produced in food or the evaluation of the metabolism of living organisms.

\section{Introduction}

Formaldehyde (FA) is highly toxic and one of the most well-known volatile organic compounds (VOCs). It is widely found in indoor living or working environments. ${ }^{(1-4)}$ FA is also produced in natural processes. For example, it is a natural metabolite of living organisms and is found in bodily fluids, tissues, and cells. ${ }^{(5,6)}$ Some foods are known to contain naturally occurring FA. For example, marine fishes and crustaceans are reported to develop FA after death, and it is accumulated during frozen storage. ${ }^{(7)}$ According to the World Health Organization (WHO), typical FA concentrations are $3-60 \mathrm{mg} \cdot \mathrm{kg}^{-1}$ in fruits and vegetables, $1 \mathrm{mg} \cdot \mathrm{kg}^{-1}$ in milk and milk products, $6-20 \mathrm{mg} \cdot \mathrm{kg}^{-1}$ in meat and fish, and 1-100 mg. $\mathrm{kg}^{-1}$ in shellfish. ${ }^{(8)}$ The International Agency for Research on Cancer (IARC) concluded that FA can be carcinogenic to humans. Therefore, the United States

"Corresponding author: e-mail: m.bdi@tmd.ac.jp

†Present address: Department of Electronics and Bioinformatics, School of Science and Technology, Meiji University, 1-1-1 Higashi-Mita, Kawasaki, Kanagawa 214-8571, Japan

ISSN 0914-4935 @ MYU K.K. 
Environment Protection Agency (EPA) set a guideline for an acceptable daily intake of FA of 0.2 $\mathrm{mg} \cdot \mathrm{kg}^{-1}$ of body weight.

There have been a number of efforts to develop sensors to detect gaseous FA. Chung et al. fabricated core-shell structures to enable the measurement of gaseous FA at room temperature. ${ }^{(9)}$ Wang et al. introduced a colorimetric method that employed a methyl yellow-impregnated nanofiber/net to improve response time and sensitivity.(10) Methods for measuring FA concentration in food have also been reported. They include colorimetric methods using Nash reagent for maple syrup $^{(11)}$ and gas chromatography (GC) or high-performance liquid chromatography (HPLC) for milk, ${ }^{(12)}$ fish, ${ }^{(13,14)}$ and coffee. ${ }^{(15)}$ Despite their advantages, these methods require laborious and complicated processes to obtain results, and often samples are destroyed for measurement. In addition, for GC or HPLC, specificity is a problem in the analysis of complex compounds.

Recently, we have introduced a fiber-optic biochemical gas sensor (bio-sniffer) that exploits enzyme-based molecular recognition. ${ }^{(16-21)}$ In the bio-sniffer, FA is detected via fluorescence emitted from the reduced form of $\beta$-nicotinamide adenine dinucleotide (NADH) that is produced through enzymatic reaction with formaldehyde dehydrogenase (FALDH). The fiber is used to excite NADH and collect the fluorescence; thus, the system is simple and easy to handle. Furthermore, because fluorescence is emitted instantaneously as a response to gaseous FA, real-time monitoring can be performed. Previously, using the bio-sniffer, we have demonstrated the detection and real-time monitoring of gaseous FA at low ppb levels. ${ }^{(16)}$

Although the bio-sniffer showed high sensitivity and selectivity in previous reports, these characteristics were assessed only with pristine standard samples, i.e., FA gas produced by a standard gas generator. However, a real sample is generally complex, which makes it more challenging for the sensor to measure such a sample. In particular, gas released from living organisms is found in trace amounts and mixed with many different compounds. Therefore, in this study, we demonstrate the capability of the bio-sniffer to directly and nondestructively measure FA gas in naturally produced complex gas from a real sample (food). A Shiitake mushroom (Lentinus edodes) was chosen as a model sample because the mushroom produces FA as well as pyruvic acid and sulfur compounds through an enzymatic reaction that forms lenthionine-a compound responsible for the flavor of Shiitake. ${ }^{(22-26)}$ First, the bio-sniffer for standard FA vapor was characterized to assess the capability of continuous monitoring and sensor sensitivity. Then, a gas mixture containing FA released from the mushroom was measured using the bio-sniffer. To verify the results from the bio-sniffer, mushrooms were prepared in three different states-dried (DRY), rehydrated with deionized water (W-RH), and with $\gamma$-GTPase inhibitor (I-RH), and the concentration differences in gaseous FA were compared.

\section{Materials and Methods}

\subsection{Reagents and chemicals}

An enzyme, FALDH, from Pseudomonas sp. with an activity of 1 unit $\mathrm{mg}^{-1}$ was purchased from Funakoshi (Japan). An 80- $\mu$ m-thick hydrophilic polytetrafluoroethylene (H-PTFE) filter with $80 \%$ porosity and a pore size of $0.2 \mu \mathrm{m}$ was used for an enzyme membrane [Millipore (USA)]. A polymer immobilizing FALDH in the membrane, poly[2-methacryloyloxyethyl phosphorylcholine (MPC)-co-2-ethylhexyl methacrylate (EHMA)] (PMEH), was synthesized in house by a free- 
radical polymerization method. ${ }^{(20)} \mathrm{NADH}$ and $\mathrm{NAD}^{+}$(the oxidized form of $\beta-\mathrm{NADH}$ ) were from Oriental Yeast (Japan). Phosphate buffer (PB, $80 \mathrm{mM}$, pH 8.0) solution containing $20 \mathrm{mM} \mathrm{NAD}^{+}$ $\left(\mathrm{PB} / \mathrm{NAD}^{+}\right)$was also prepared. Dried Shiitake mushrooms (Japan) were purchased at a local supermarket.

\subsection{Construction of the bio-sniffer}

The enzyme immobilized membrane was prepared as described in detail in our previous work. ${ }^{27,28)}$ Briefly, FALDH/PB solution was mixed with PMEH/ethanol solution so that eventually their concentrations were 50 units $\cdot \mathrm{cm}^{-2}$ and $1 \mathrm{~mL} \cdot \mathrm{cm}^{-2}$ on the membrane, respectively. Afterwards, the H-PTFE membrane was coated with the mixed solution, followed by curing in a refrigerator at $4{ }^{\circ} \mathrm{C}$ for 180 min. Finally, excess FALDH was rinsed off with PB.

The detection principle of the optode in the bio-sniffer and its sensing region are shown in Fig. 1. A flow cell, through which $\mathrm{PB} / \mathrm{NAD}^{+}$flows, is attached over the optical fiber end. On the top of the flow cell, the enzyme membrane is tightly fixed with a silicone O-ring. When FA reaches the membrane, it is easily dissolved into the membrane, leading to the enzymatic reaction accompanied by the reduction of $\mathrm{NAD}^{+}$as described in Fig. 1. By employing a PB circulation system, the resultant NADH is immediately washed away from the sensing region after the fluorescence emission; thus, real-time monitoring can be achieved. The circulation also benefits by avoiding enzyme deactivation and from removing the products and the excess substrates from the reaction. As a result, the sensing region is always refreshed, and the output fluorescence signal of the biosniffer is determined by a trade-off between the production rate (FA concentration) and the removal rate of $\mathrm{NADH}$ (flow rate of $\mathrm{PB}$ ).
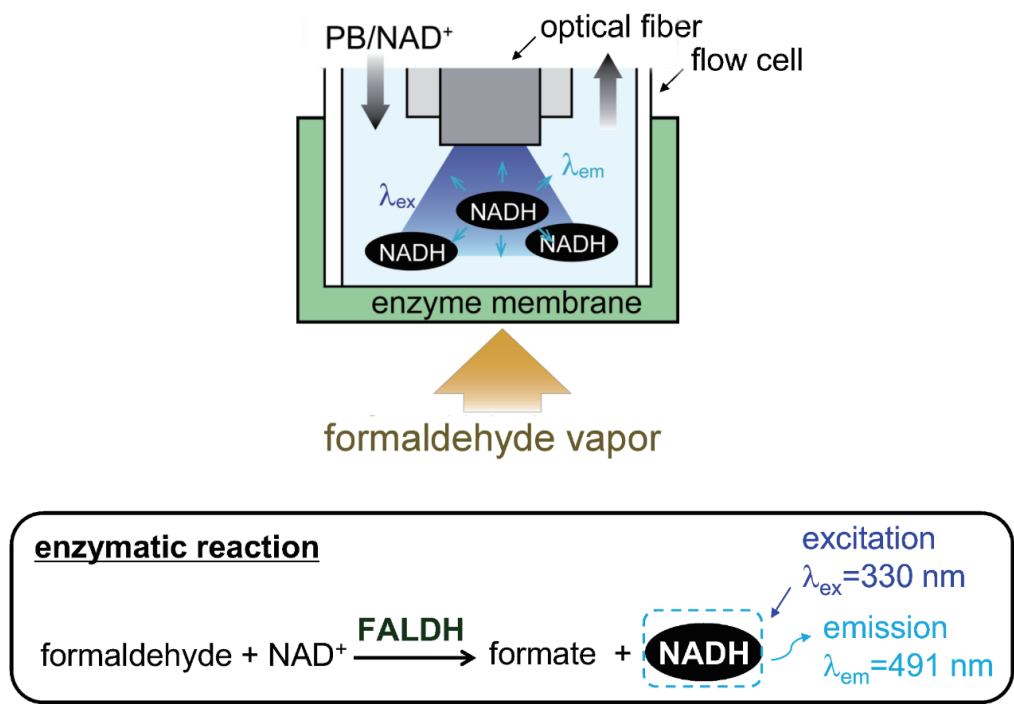

Fig. 1. (Color online) Schematic diagram of the optode in the bio-sniffer and the reaction scheme of FALDHmediated enzymatic reaction to produce NADH. NAD ${ }^{+}$-containing PB solution is flowed between a fiber-optic end and FALDH immobilized membrane. The enzymatic reaction occurs when FA reaches the membrane. The resultant NADH is excited by UV light from the optical fiber and the emitted fluorescence is detected. 
In Fig. 2, the setup of the bio-sniffer is shown. As a light source, an array of four ultraviolet light-emitting diodes arranged in parallel (multi-UV-LED) was developed in house. UV light emitted from the multi-UV-LED is coupled into one branch (core diameter of $0.4 \mathrm{~mm}$ ) of a dualbranch optical fiber bundle (KLV, Japan) and propagates toward the fiber end of the optode. When NADH is produced through the enzymatic reaction with FA at the enzyme membrane, the fluorescence $\left(\lambda_{\mathrm{em}}=491 \mathrm{~nm}\right)$ emitted from NADH excited by the UV light $\left(\lambda_{\mathrm{ex}}=330 \mathrm{~nm}\right)$ is coupled back to the fiber bundle and guided through the other branch (core diameter of $0.6 \mathrm{~mm}$ ) to a photomultiplier tube (PMT, Hamamatsu Photonics, Japan). In the setup, two different band pass filters (BPs) were placed after the multi-UV-LED and before PMT to eliminate unwanted light.

\subsection{Characterization of the bio-sniffer}

A calibration curve for standard gaseous FA was determined with the setup depicted in Fig. 2(a). A custom-made standard gas generator (Gastec, Japan) was used as a source of FA; the generator was equipped with a large-volume thermostat bath to generate highly diluted FA vapor down to a level of 20 ppt. For FA vapor concentration higher than $30 \mathrm{ppb}$, we used a commercialized standard gas generator (PD-1B, Gastec, Japan). The generators were also able to produce and dispense high-purity air that was prepared by filtering out unwanted compounds including FA. During the experiment, the flow rates of $\mathrm{PB} / \mathrm{NAD}^{+}$and $\mathrm{FA}$ vapour or air were fixed at 1.5 and $200 \mathrm{~mL} \cdot \mathrm{min}^{-1}$, respectively.

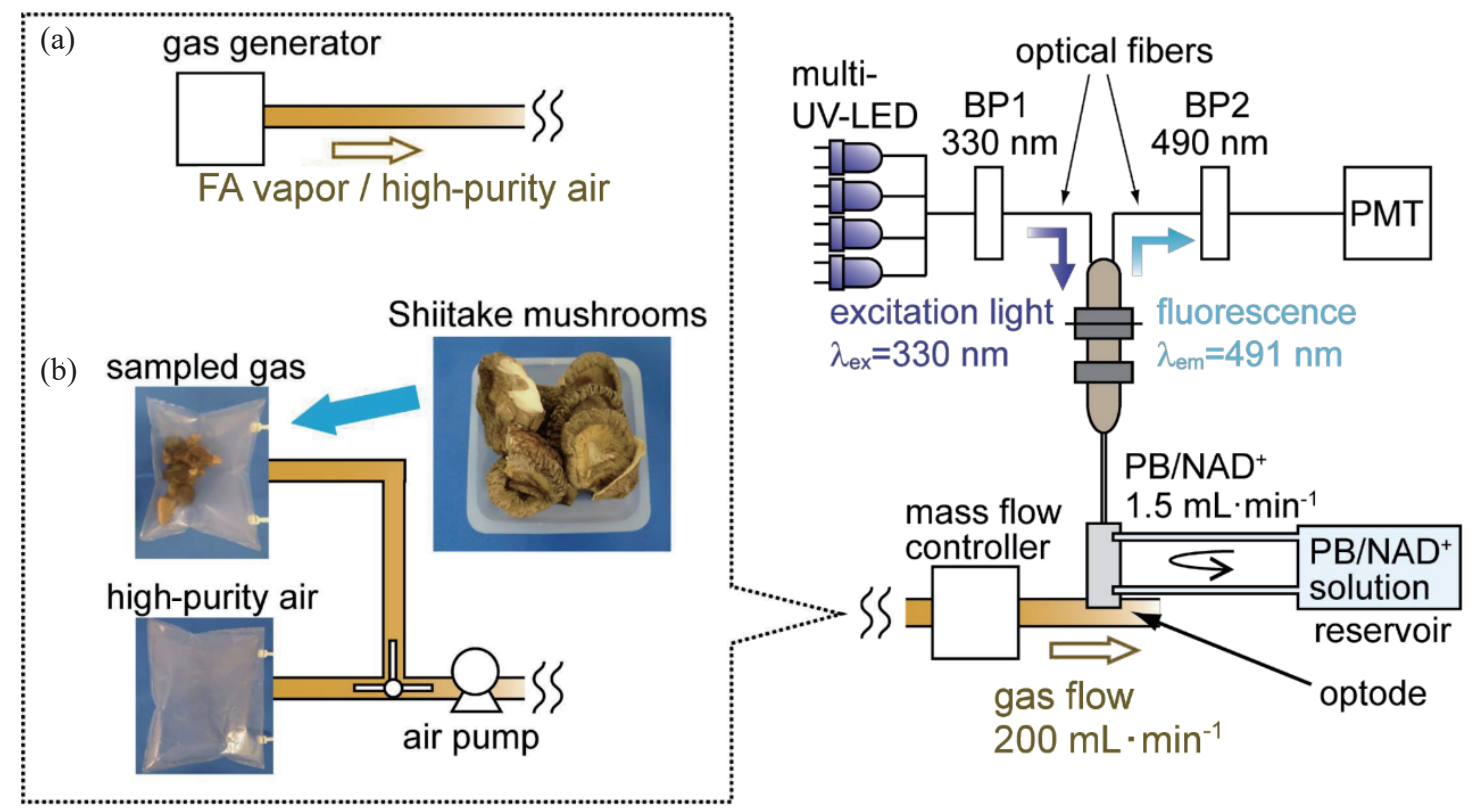

Fig. 2. (Color online) Experimental setup for (a) the characterization of the bio-sniffer using standard FA vapor sample and (b) assessment of the ability to directly measure FA gas released from Shiitake mushrooms. In both experiments, high-purity air was used as a reference. The resultant NADH is excited with UV light irradiated from the end of the optical fiber bundle. The emitted fluorescence is coupled back to the fiber, guided to PMT. BP, band pass filter; PMT, photomultiplier tube; PB, phosphate buffer. 


\subsection{Detection of FA from food sample (Shiitake mushrooms)}

Shiitake mushrooms in three different states-DRY, W-RH, and I-RH-were prepared to verify the bio-sniffer by comparing with a previous work. ${ }^{(29)}$ In the production of FA by the Shiitake mushrooms, $\gamma$-GTPase plays an important role because FA is produced in two enzymatic cascades beginning with $\gamma$-GTPase. ${ }^{(29)}$ In the first reaction, neither FA nor des-glu lenthinic acid is produced without $\gamma$-GTPase activity. Then FA is also not produced in the second reaction with C-S lyase because there is no des-glu lenthinic acid (see in Fig. 3). Therefore, we used I-RH as a control, and decreased FA emission from the mushroom was expected. The inhibitor was a serineborate complex prepared by mixing borate, L-serine, D-serine, and $\alpha$-methyl-DL-serine at a final concentration of $50 \mathrm{mM}$ and $\mathrm{pH} 7.0$.

The setup employing a batch flow measurement system for detecting FA from Shiitake mushroom is presented in Fig. 2(b). The gas from DRY was obtained by keeping $20 \mathrm{~g}$ of dried mushrooms in a $20 \mathrm{~L}$ sampling bag filled with high-purity air for $2 \mathrm{~h}$ at $25^{\circ} \mathrm{C}$. For the rehydrated mushrooms, prior to keeping them in bags, the dried mushrooms were soaked in deionized water (for W-RH) or $\gamma$-GTPase inhibitor (for I-RH) for $1 \mathrm{~h}$. The bag with only air was also prepared as a blank sample. After sampling the gas, the bag was connected to a tube to direct the gas towards the optode of the bio-sniffer. While conducting the experiments, the flow rates of $\mathrm{PB} / \mathrm{NAD}^{+}$and the sample gases were fixed at 1.5 and $200 \mathrm{~mL} \cdot \mathrm{min}^{-1}$, respectively, as was the case described in Sect. 2.3.

\section{Results and Discussion}

\subsection{Characterization of the bio-sniffer}

First, the characteristics of the bio-sniffer for FA vapor were investigated with standard gaseous FA. A representative fluorescence intensity change $(\Delta F I)$ upon exposure to $10 \mathrm{ppb} F A$ is shown in Fig. 4. When the FA vapor was introduced, the fluorescence intensity (FI) increased rapidly as a result of the production of NADH by the enzymatic reaction in the membrane. The response intensity change $\Delta F I$ reached $90 \%$ of saturation about $2.5 \mathrm{~min}$ after the introduction of FA. At saturation, production and removal rates of NADH were balanced. When changing to high-purity air after 10

\section{FA production in Shiitake mushroom

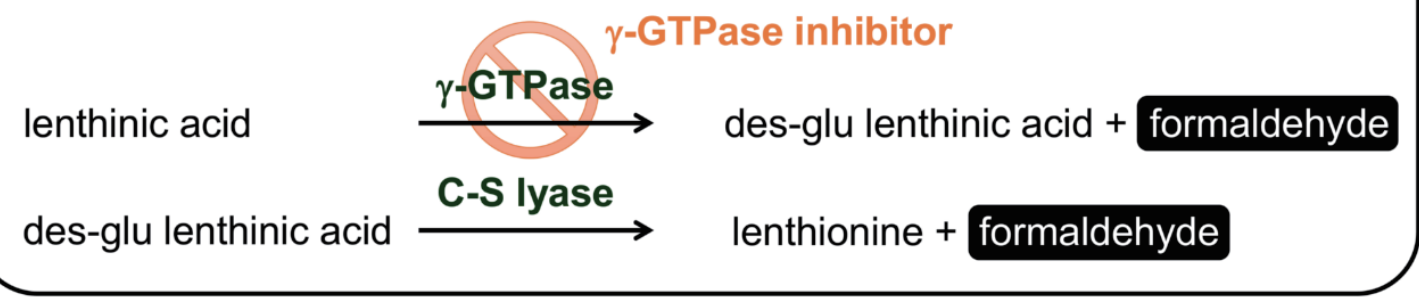

Fig. 3. (Color online) Production of FA in Shiitake mushrooms. $\gamma$-GTPase inhibitor refrains from the enzymatic reactions with $\gamma$-GTPase, and thereby the subsequent reaction with C-S lyase does not occur. As a result, little FA production is expected. 


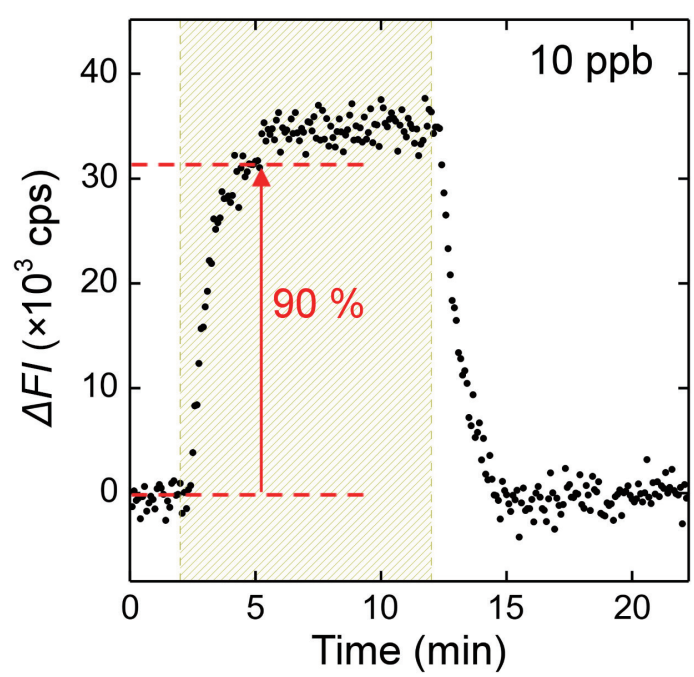

Fig. 4. (Color online) Representative $\triangle F I$ as a response to standard FA vapor sample. FA concentration was fixed at $10 \mathrm{ppb}$. The hatched region indicates a flow time of $10 \mathrm{~min}$.

min FA flow, PB washed away NADH from the sensing region, at which point the intensity dropped to the level of the initial intensity. It took 2 min for recovery to $10 \%$ saturation.

The calibration curve for various FA concentrations was obtained as shown in Fig. 4. The error bars represent the standard deviations of 5 measurements. A fitting curve is obtained for concentrations from 0.6 to $500 \mathrm{ppb}$, which can be expressed by the equation: $\Delta F I$ (cps) $=2519.2 \times$ $[\mathrm{FA}]^{0.87}$ with a correlation coefficient of 0.998 , where $[\mathrm{FA}(\mathrm{ppb})]$ denotes the FA concentration in ppb.

The limit of detection (LOD) of the bio-sniffer was $1.1 \mathrm{ppb}$, which was determined at the FA concentration where three times the standard deviation $3 \sigma=2718$ counts per second (cps) for a blank sample (high-purity air) equalled $\Delta F I$. According to our previous study, the bio-sniffer showed no response to acetaldehyde, acetone, benzene, methanol, or ethanol, while fully responding to FA because of the high specificity of FALDH. ${ }^{(16)}$ The ability to continuously measure FA vapor was also examined using 5 measurements at $30 \mathrm{ppb}$ for $110 \mathrm{~min}$ in total. The high reproducibility of the bio-sniffer was demonstrated using a coefficient of variation (C.V.) of 3.4\% (see the inset in Fig. 5).

\subsection{Detection of FA from food sample (Shiitake mushrooms)}

Finally, we measured FA released from food samples (Shiitake mushrooms) using the bio-sniffer. In Fig. 6, the sensor responses to the gas mixture collected from DRY and W-RH are presented. FI immediately increased from the baseline upon introduction of both gaseous samples. This result confirms that the gas contained FA. $\triangle F I$ for DRY reached $1.5 \times 10^{4} \mathrm{cps}$, which corresponds to an FA concentration of $7.9 \mathrm{ppb}$ according to the calibration curve. Assuming that the released FA level correlated to an FA concentration in the mushrooms of $110-320 \mathrm{mg} \cdot \mathrm{kg}^{-1},{ }^{(30)}$ the bio-sniffer could directly detect FA at about one order of magnitude lower concentration in food, including vegetable, fruit or shellfish. 


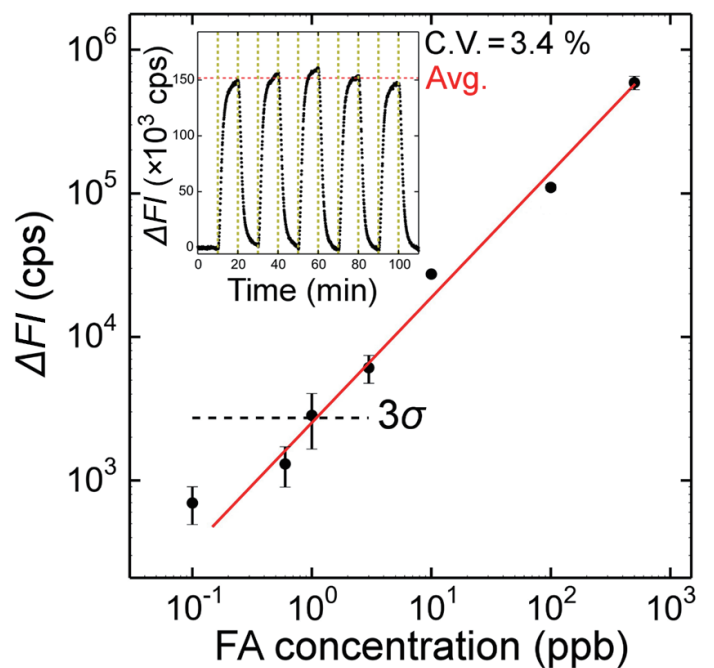

Fig. 5. (Color online) Calibration curve for the bio-sniffer for standard FA vapor. The dashed line indicates that the signal equals 3 times the noise $\sigma$ at which the LOD of $1.1 \mathrm{ppb}$ is determined. The inset shows continuous monitoring of FA at the fixed concentration of $30 \mathrm{ppb}$ with C.V. of $3.4 \%$.

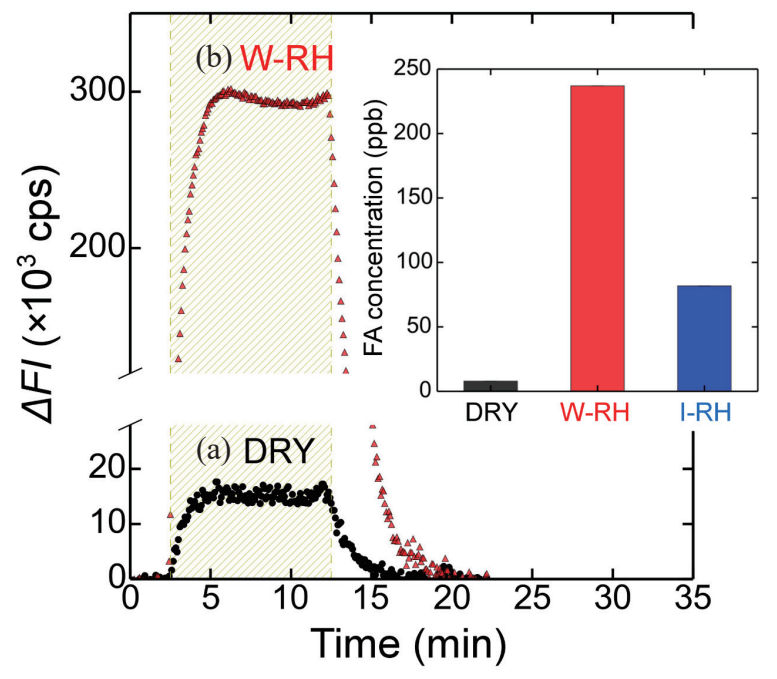

Fig. 6. (Color online) Real-time fluorescence response of the bio-sniffer upon introduction of gas collected from (a) DRY and (b) W-RH. The hatched region indicates the flow time of $10 \mathrm{~min}$. The inset shows a comparison of FA concentrations determined for DRY, W-RH, and I-RH.

For W-RH, FI was changed dramatically and reached $2.9 \times 10^{5} \mathrm{cps}$. The corresponding FA concentration was $237.0 \mathrm{ppb}$, which was 30-fold higher than that from DRY. These results indicate that FA detected in the experiments was generated endogenously as a result of rehydration.

The comparison of FA concentration determined for each sample is shown in the inset in Fig. 6. The FA concentration of I-RH was $81.8 \mathrm{ppb}$, which was lower than that of W-RH since the activity of $\gamma$-GTPase was inhibited by the serine-borate complex. ${ }^{(31)}$ These trends are in good agreement with previously measured FA production. ${ }^{(29)}$

\section{Conclusions}

In this paper, we examined the ability of the bio-sniffer to directly and nondestructively measure a gaseous FA released from food (Shiitake mushrooms). The mushrooms in three different states (DRY, W-RH, and I-RH) were prepared to compare each FA level. W-RH exhibited the highest FA concentration due to endogenously produced FA after rehydration. In contrast, FA concentration from I-RH was less than half of the W-RH. This result was attributed to inhibiting the activity of an enzyme ( $\gamma$-GTPase) that is needed to produce FA in the mushroom. From the clear response observed for DRY and the determined LOD (1.1 ppb), we anticipate that the bio-sniffer will be able to directly measure FA released from other foods, such as vegetables, fruits or shellfish that contain about an order of magnitude lower concentration of FA than dried Shiitake mushroom. 


\section{Acknowledgements}

This work was supported by the Japan Society for the Promotion of Science (JSPS) Grant-in-Aid for Scientific Research System, the Japan Science and Technology Agency (JST), and the Ministry of Education, Culture, Sports, Science and Technology (MEXT) Special Funds for Education and Research "Advanced Reasearch Program in Neo-Biology".

\section{References}

1 S. Colak and G. Colakoglu: Build. Environ. 39 (2004) 533.

2 S. Costa, P. Coelho, C. Costa, S. Silva, O. Mayan, L. S. Santos, J. Gaspar, and J. P. Teixeira: Toxicology 252 (2008) 40.

3 S. Takahashi, K. Tsuji, K. Fujii, F. Okazaki, T. Takigawa, A. Ohtsuka, and K. Iwatsuki: J. Dermatol. 34 (2007) 283.

4 R. Wiglusz, E. Sitko, G. Nikel, I. Jarnuszkiewicz, and B. Igielska: Build. Environ. 37 (2002) 41.

5 P. H. Yu, B. A. Davis, and Y. Deng: Biochem. Pharmacol. 61 (2001) 741.

6 P. H. Yu, C. T. Lai, and D. M. Zuo: Neurochem. Res. 22 (1997) 615.

7 M. Careche and M. Tejada: Z. Lebensm. Unters. Forsch. 193 (1991) 533.

8 WHO Regional Office for Europe: Air Quality Guidelines - Second Edition Chapter 5.8 Formaldehyde, 2001.

9 F.-C. Chung, R.-J. Wu, and F.-C. Cheng: Sens. Actuators, B 190 (2014) 1.

10 X. Wang, Y. Si, J. Wang, B. Ding, J. Yu, and S. S. Al-Deyab: Sens. Actuators, B 163 (2012) 186.

11 K. Helrich, ed.: Official Methods of Analysis of the Association of Official Analytical Chemists in Association of Official Analytical Chemist, 15th ed. (Arlington, VA, 1990).

12 J. Kaminski, A. S. Atwal, and S. Mahadevan: J. Liq. Chromatogr. 16 (1993) 521.

13 A. Yasuhara and T. Shibamoto: J. Agric. Food Chem. 43 (1995) 94.

14 M. Cristina, V. Mota, G. V. Santos, and J. B. De Andrade: J. Chromatogr. Sci. 39 (2001) 173.

15 T. Hayashi, C. A. Reece, and T. Shibamoto: J. Assoc. Off. Anal. Chem. 69 (1986) 101.

16 H. Kudo, Y. Suzuki, T. Gessei, D. Takahashi, T. Arakawa, and K. Mitsubayashi: Biosens. Bioelectron. 26 (2010) 854.

17 H. Saito, Y. Kaneko, Y. Hashimoto, T. Shirai, H. Kudo, K. Otsuka, and K. Mitsubayashi: Sens. Actuators, B 123 (2007) 877.

18 K. Mitsubayashi, H. Matsunaga, G. Nishio, S. Toda, Y. Nakanishi, H. Saito, M. Ogawa, and K. Otsuka: Sens. Actuators, B 108 (2005) 660.

19 K. Mitsubayashi, H. Matsunaga, G. Nishio, S. Toda, and Y. Nakanishi: Biosens. Bioelectron. 20 (2005) 1573.

20 H. Kudo, M. Sawai, Y. Suzuki, X. Wang, T. Gessei, D. Takahashi, T. Arakawa, and K. Mitsubayashi: Sens. Actuators, B 147 (2010) 676.

21 H. Kudo, T. Goto, T. Saito, H. Saito, K. Otsuka, and K. Mitsubayashi: Microchim. Acta 160 (2007) 421.

22 K. Yasumoto, K. Iwami, and H. Mitsuda: Agric. Biol. Chem. 35 (1971) 2070.

23 K. Yasumoto, K. Iwami, and H. Mitsuda: Agric. Biol. Chem. 35 (1971) 2059.

24 S. Okada, S. Iga, and H. Isaka: J. Hyg. Chem. 18 (1972) 353.

25 Y. Liu, Y. Yuan, X. Lei, H. Yang, S. A. Ibrahim, and W. Huang: Food Chem. 138 (2013) 2174.

26 A. C. De Groot, M. Flyvholm, G. Lensen, T. Menn, and P.-J. Coenraads: Contact Dermatitis 61 (2009) 63.

27 H. Kudo, M. Sawai, X. Wang, T. Gessei, T. Koshida, K. Miyajima, H. Saito, and K. Mitsubayashi: Sens. Actuators, B 141 (2009) 20.

28 H. Kudo, T. Yagi, M. X. Chu, H. Saito, N. Morimoto, Y. Iwasaki, K. Akiyoshi, and K. Mitsubayashi: Anal. Bioanal. Chem. 391 (2008) 1269.

29 K. Iwami, K. Yasumoto, and H. Mitsuda: J. Jpn. Soc. Nutr. Food Sci. 27 (1974) 393.

30 D. J. Mason, M. D. Sykes, S. W. Panton, and E. H. Rippon: Food Addit. Contam. 21 (2004) 1071.

31 S. S. Tate and A. Meister: Proc. Natl. Acad. Sci. 75 (1978) 4806. 